\title{
One-Step Surgical Procedure for the Treatment of Osteochondral Defects with Adipose-Derived Stem Cells in a Caprine Knee Defect: A Pilot Study
}

\author{
Wouter J.F.M. Jurgens, ${ }^{1,2}$ Robert Jan Kroeze, ${ }^{2,3}$ Behrouz Zandieh-Doulabi, ${ }^{2,4}$ Annemieke van Dijk, \\ Greetje A.P. Renders, ${ }^{2,4}$ Theo H. Smit, ${ }^{2,3}$ Florine J. van Milligen,, ${ }^{2,}$ \\ Marco J.P.F. Ritt, ${ }^{1,2}$ and Marco N. Helder ${ }^{2,3}$
}

\begin{abstract}
Regenerative therapies offer attractive alternatives for the treatment of osteochondral defects. Adipose-derived stromal vascular fraction (SVF) cells allow the development of one-step surgical procedures by their abundant availability and high frequency. In this pilot study we evaluated the in vivo safety, feasibility, and efficacy of this concept using scaffolds seeded with freshly isolated (SVF) or cultured adipose stem cells (ASCs), and compared these to their acellular counterparts. Osteochondral defects were created in medial condyles and trochlear grooves in knees of eight goats. Defects were filled with acellular collagen I/III scaffolds or scaffolds seeded with SVF cells or cultured ASCs. Osteochondral regeneration was evaluated after 1 and 4 months by macroscopy, immunohistochemistry, biomechanical analysis, microCT analysis, and biochemistry. After 1 month, no adverse effects were noted. Microscopic, but not macroscopic evaluation showed considerable yet not significant differences, with cell-loaded constructs showing more extensive regeneration. After 4 months, acellular constructs displayed increased regeneration, however, to a lesser degree than cell-treated constructs. The latter exhibited more extensive collagen type II, hyaline-like cartilage, and higher elastic moduli, and their glycosaminoglycan content in the cartilaginous layer better approached native tissue values. Moreover, their defect regions contained higher levels of regenerated, mature subchondral bone with more intense collagen type I staining. SVF cells tended to perform best on all parameters. In summary, this pilot study demonstrated the preclinical safety and feasibility of a one-step surgical procedure for osteochondral defect regeneration. Similar regeneration was found between freshly isolated SVF cells and cultured ASCs. Larger studies with longer follow-up are required to substantiate these findings.
\end{abstract}

Key words: adipose tissue; caprine; cartilage repair; in vivo; mesenchymal stem cells; osteochondral

\section{Introduction}

$\mathbf{T}$ ISSUE ENGINEERING THERAPIES are being evaluated as promising alternatives to heal (osteo)chondral defects. These defects have limited self-regenerative potential or result in suboptimal functional repair when using conventional joint resurfacing treatments. ${ }^{1-4}$ Adipose tissue is an attractive source of stem cells for these regenerative therapies due to its abundance and high frequency of stem cells with multiline- age differentiation capacity. ${ }^{5}$ The stem cells reside in a supportive stromal vascular fraction (SVF) that can be easily isolated. Next to the adipose stem cells (ASC), the SVF of adipose tissue consists of a heterogeneous mixture of cells, including endothelial cells, smooth muscle cells, pericytes, leucocytes, mast cells, and pre-adipocytes. ${ }^{5-7}$ Currently, most therapies focus on the use of cultured cells of various origins to obtain sufficient quantities needed for regeneration. ${ }^{3,8-12}$ The use of adipose tissue can overcome this

Departments of ${ }^{1}$ Plastic, Reconstructive, \& Hand Surgery, ${ }^{3}$ Orthopedic Surgery, and ${ }^{5}$ Pathology, VU University Medical Center (VUmc), Amsterdam, The Netherlands.

${ }^{2}$ MOVE/Skeletal Tissue Engineering Group Amsterdam (STEGA), Amsterdam, The Netherlands.

${ }^{4}$ Department of Oral Cell Biology and Functional Anatomy, Academic Center for Dentistry Amsterdam (ACTA), University of Amsterdam and VU University, Amsterdam, The Netherlands.

*Present address: Sanquin Research, Amsterdam, The Netherlands. 
culturing step since a clinically relevant number of stem cells can be isolated, creating the possibility to develop a one-step surgical procedure for the treatment of osteochondral defects. ${ }^{13}$ This concept has already been tested in vitro and showed promising results, demonstrating the ability of the ASC fraction within the SVF to attach to a scaffold material in sufficient quantities in a short time frame ( $\sim 10 \mathrm{~min})$, and the capacity to differentiate into the osteogenic and chondrogenic lineage. ${ }^{14,15}$ Obvious advantages of this approach in humans are its patient friendliness and its lower costs, in addition to avoidance of a second surgical intervention and expensive in vitro culturing steps.

To further investigate the safety and feasibility of this onestep surgical procedure for the treatment of osteochondral defects, we employed an in vivo goat model. This large animal model is known for its suitability to evaluate osteochondral defects and its possible translation to the human situation. ${ }^{16,17}$ To evaluate the potential modulatory effects of other cell types present in the SVF, scaffolds seeded with freshly isolated adipose-derived stromal cells (containing other cell types) were compared with scaffolds seeded with adipose-derived stem cells cultured to homogeneity (i.e., devoid of other cell types). ${ }^{14,15}$ Bare, acellular scaffolds were introduced as negative control.

\section{Materials and Methods}

\section{Experimental animals}

Eight skeletally mature female Dutch milk goats (average body weight: $82.4 \pm 11.7 \mathrm{~kg}$ ) were used in this study. Protocols were approved by both a Scientific Board as well as the Animal Ethics Committee of the VUmc.

\section{Surgical set-up}

Animals were considered healthy based on physical examination and viral and bacterial tests. Anesthesia was induced with an intravenous combination of $10 \mathrm{mg}$ ketamine (Alfasan, Woerden, The Netherlands), $1.5 \mathrm{mg}$ atropine (Pharmachemie, Haarlem, The Netherlands), and 10-20 mg Etomidate (B. Braun, Melsungen, Germany) per $\mathrm{kg}$ of body weight. After endotracheal intubation, goats received a bolus of $15 \mathrm{mg}$ of midazolam intravenously (IV; Dormicum, Actavis, Baarn, The Netherlands), which was repeated if indicated. Anesthesia was maintained with $1 \%-2 \%$ isoflurane per inhalation. Analgesia consisted of $250 \mathrm{~mL}$ of fentanyl IV (Hameln Pharmaceuticals, Hameln, Germany) perioperatively and buprenorfine intramuscularly (Temgesic, Schering-Plough, Utrecht, The Netherlands) postoperatively and was maintained for 7 days with Novum 20 subcutaneously (Meloxicam, Boehringer Ingelheim, Alkmaar, The Netherlands). Penicillin and streptomycin were administered as perioperative antibiotics.

All goats underwent surgery twice: during the first procedure adipose tissue was harvested from the left thoracolumbar vertebrae $( \pm 30 \mathrm{~g})$ and processed for SVF. This was subsequently cultured to obtain a homogenous population of ASC. After surgery goats were allowed to move freely. Two weeks later, adipose tissue was harvested from the right thoracolumbar region, to procure freshly isolated SVF. This second harvesting/processing step was followed by implantation surgery.

\section{Adipose-derived stem cell procurement and culturing}

SVF was isolated from each goat as described previously. ${ }^{18,19}$ In short, adipose tissue was dissociated by using $0.5 \mathrm{U} / \mathrm{mL}$ Liberase Blendzyme 3 (Roche Diagnostics, Almere, The Netherlands) and filtered, and the resulting single-cell suspension was pelleted. The remaining SVF was either implanted directly in the one-step surgical procedure (surgery 2), or used for culturing to obtain a homogeneous population of ASC (surgery 1). A small fraction of each SVF was used for colony-forming unit assays to determine the percentage of stem cells within the preparations. For culturing, SVF was plated in $25-\mathrm{cm}^{2}$ tissue culture flasks. Medium consisted of Dulbecco's modified Eagle's medium (DMEM; Gibco, Paisley, United Kingdom) supplemented with $10 \%$ fetal bovine serum (FBS; Hyclone, Logan, UT), $100 \mathrm{U} / \mathrm{mL}$ penicillin, $100 \mu \mathrm{g} / \mathrm{mL}$ streptomycin and $2 \mathrm{mM}$ L-glutamine (all Invitrogen, Gibco, Bleiswijk, Netherlands). Cultures were grown in a humidified incubator at $37^{\circ} \mathrm{C}$ in an atmosphere of $5 \%$ $\mathrm{CO}_{2}$. When reaching $80 \%-90 \%$ confluency, cells were detached with $0.5 \mathrm{mM}$ EDTA/0.05\% trypsin (Invitrogen) for $5 \mathrm{~min}$ at $37^{\circ} \mathrm{C}$ and replated. In this way a homogeneous population of ASCs was obtained. Cultured ASCs were used for implantation at passage 3 in surgery 2 .

\section{Colony-forming unit-fibroblast assays}

Colony-forming unit-fibroblast assays (CFU-f assays) were performed as described previously. ${ }^{19}$ In short, two sixwell plates were prepared with $10^{4}$ nucleated SVF cells in the upper and $10^{3}$ cells in the lower row, respectively. Colony formation was allowed for 7-10 days, depending on number and growth kinetics of the colonies (merging of colonies was avoided). After fixation, colonies were stained with $0.2 \%$ toluidine blue solution in borax buffer and counted.

\section{Implant preparation}

Treatment groups existed of phosphate-buffered saline (PBS), freshly isolated SVF $\left(5 \times 10^{6}\right.$ cells $)$, and cultured ASCs $\left(5 \times 10^{5}\right.$ cells $)$. The three different mixtures were seeded in $70 \mu \mathrm{L}$ onto a $5 \mathrm{~mm}$ diameter $\times 3 \mathrm{~mm}$ high collagen type I/III scaffold (Optimaix ${ }^{\circledR}$, Matricel GmbH, Herzogenrath, Germany), which was shown to allow chondrogenic differentiation in vitro, ${ }^{15}$ and after $30 \mathrm{~min}$ implanted into the defect.

\section{Implantation surgery}

Animals were prepared for surgery as already described. A medial parapatellar incision was made to expose the right knee joint and to create two reproducible fresh critically sized osteochondral defects (diameter $5 \mathrm{~mm}$, depth $3.5 \mathrm{~mm})^{16,20}$ in the right trochlea femoris and the right medial femoral condyle (four in total per animal) using a custommade grinder and holder. According to the randomization schedule, each goat should have been treated with one or two (randomized per each two goats) SVF and ASC-loaded scaffolds, and one acellular control. However, due to low cell yields from two goats, in total 11 SVF- and 12 ASCtreated constructs and nine acellular scaffolds were implanted. Scaffolds recessed just under the articular surface to minimize early shear stress. A small drop of synovial fluid was added to induce swelling and press-fitting of the implant in the defect site. For additional retainment, Prolene ${ }^{\circledR}$ 5-0 cross-suturing 
was applied. Incisions were closed in layers using Vicryl ${ }^{\circledR}$ 2-0 sutures (Ethicon, Leiden, Netherlands).

A full-limb cast $\left(\right.$ Softcast ${ }^{\circledR}, 3 \mathrm{M}$, Zoeterwoude, The Netherlands) was applied for 1 month, restricting the range of motion to about $5^{\circ} .{ }^{21}$ This caused only minor restriction of the gait, but did not affect the weight-bearing function of the limb. After 1 month, two goats (containing three SVF and ASC-loaded scaffolds and two acellular scaffolds), were sacrificed using a lethal dose of pentobarbitalnatrium (Euthasol 20\%, Ast Beheer, Oudewater, The Netherlands) to analyze any adverse effects. In the remaining six goats free movement for the ensuing 12 weeks was allowed before sacrifice and analysis of midterm osteochondral regeneration. Since this pilot study was primarily designed to investigate safety and feasibility with a small number of animals, no long-term repair was investigated.

\section{Sample preparation and assessment}

Upon sacrifice, gross resemblance of the newly formed tissue in the defects was scored using the method of Moran et al. ${ }^{22}$ The 1-month samples were processed for histology only to demonstrate any adverse effects, whereas the 4month samples were first analyzed mechanically, followed by splitting the specimens into two equal parts. One part was used for microCT analysis followed by histology, whereas the other part was processed for biochemistry (SVF, $n=8$; ASC, $n=9$; acellular, $n=7$ ). Undamaged control cartilage from the same implantation site were used for comparison in biochemical, microCT, and mechanical analyses.

\section{Mechanical analysis}

Each specimen assigned for mechanical analysis was tested with a specially designed benchtop indenter. Specimens were immersed in PBS to prevent fluid loss. The measurement setoff point was determined by putting the indenter at the surface of each specimen until the stress force increased by $10 \mu \mathrm{N}$. The tensile stress-relaxation property of each specimen and its native cartilage control was determined at increasing steps of 50, 100,200 , and $300 \mu \mathrm{m}$ indentation at a constant speed of $20 \mu \mathrm{m} /$ sec with a 4-mm-diameter bold-tip probe. These indentation depths were chosen based on previous studies ${ }^{8,23,24}$ and proven reliable for determination of response force and calculation of elastic modulus in a pilot experiment (data not shown). The response force was recorded as a function of time using a stress gauge on the base of the specimen and transformed to compressive stress by Labview Software ${ }^{\circledR}$ (National Instruments, Woerden, Netherlands). Next, the elastic or Young's modulus could be calculated using Hertz equation, ${ }^{25}$ setting $v$ at $0.30,{ }^{26}$ using the following equation:

$$
F=\frac{2^{3 / 2} \times 4 R^{1 / 2}}{3\left[\left(1-v^{2}\right) / E\right]}
$$

where $F=$ force, $R$ =radius, $v=$ velocity, and $E=$ Young's modulus.

To correct for variation of implantation site, results were also depicted as percentage of each sample compared to the adjacent native cartilage tissue.

\section{Semiquantitative histological analysis}

The histological specimens were fixed in $10 \%$ formalin, decalcified in Christensens solution for 1 week, embedded in paraffin, and cut into $6-\mu \mathrm{m}$-thick sagittal sections. Sections were stained using standard hematoxylin/eosin, and adjacent sections were stained with Alcian blue $(\mathrm{pH} 2.5)$ for the detection of proteoglycans. Digital images were obtained using a Leica microscope and Leica QWin V3 software (Wetzlar, Germany). Semiquantitative histological scoring was performed according to the modified cartilage repair scoring system used by other authors, ${ }^{22,27-30}$ which has been evaluated as a reliable and reproducible scoring system. ${ }^{31}$ The following parameters were assessed: tissue hyalinity (absence of coarse collagen fibers), metachromatic staining of the matrix with Alcian blue, surface irregularity, chondrocyte clustering, regenerated subchondral bone, bonding to the adjacent articular cartilage, inflammatory cell infiltration around the implant, and freedom from degenerative changes in the adjacent cartilage, with a maximum score of 21 (best result). Assessments were performed by two independent observers that were blinded to reduce observational bias. The representative score for each parameter was determined by averaging scores of the two independent observers and reported separately under each independent parameter to be able to compare each parameter for the three different groups with the other.

\section{Immunohistochemical staining}

Expression of collagen type I and II was studied by immunohistochemical staining against collagen type I (ab23446, Abcam, Cambridge, United Kingdom) and collagen type II (II-II6B3, Developmental Studies Hybridoma Bank, Iowa City, IA). For staining of collagen type II, sections were pretreated with pronase and hyaluronidase and incubated with a 1:50 dilution of the primary antibody at $4^{\circ} \mathrm{C}$ overnight, and the presence of type II collagen was visualized by Dako REAL $^{\mathrm{TM}}$ EnVision ${ }^{\mathrm{TM}} / \mathrm{HRP}$ (Dako, Carpinteria, CA) with 3,3'-diaminobenzidine (DAB) solution as substrate. ${ }^{19}$

For staining of collagen type I, sections were pretreated with $10 \mu \mathrm{g} / \mathrm{mL}$ proteinase $\mathrm{K}$ for $30 \mathrm{~min}$, incubated with 1:100 dilution of monoclonal mouse collagen type I at $4^{\circ} \mathrm{C}$ overnight, and visualized by Dako REAL ${ }^{\mathrm{TM}}$ EnVision ${ }^{\mathrm{TM}} /$ HRP (Dako) in combination with DAB solution.

Specimens themselves served as negative controls, since collagen type 1 and 2 specifically stained in the subchondral bone and chondral part, respectively. Furthermore, samples with an IgG antibody were added to check for specificity of the Envision/HRP substrate.

\section{Contrast-enhanced microCT analysis}

A $\mu$ CT-40 system (Scanco Medical, Brüttisellen, Switzerland) was used to obtain and assess three-dimensional reconstructions of both bone and articular cartilage. Per scan, eight samples were placed in a cylindrical specimen holder (diameter $35 \mathrm{~mm}$ ) and scanned in air with a resolution of $18 \mu \mathrm{m}$ at $55 \mathrm{kV}, 177 \mu \mathrm{A}$, and $750 \mathrm{msec}$ integration time. A reservoir on the bottom of the specimen holder contained a standardized volume of $4 \mathrm{~mL}$ of PBS to prevent dehydration. All formalinfixed samples were scanned before and after a cartilage contrast-enhancement procedure. The latter procedure comprised immersion in $150 \mathrm{~mL}$ of $40 \%$ Hexabrix 320 contrast agent (Guerbet Nederland BV, The Netherlands) for $1 \mathrm{~h}$ at $37^{\circ} \mathrm{C}$, after which samples were scanned..$^{32}$ The contrast enhancement technique exploits the negative charge of sulfated glycosaminoglycans (sGAGs) within the extracellular matrix by assuming an inverse relationship between Hexabrix 


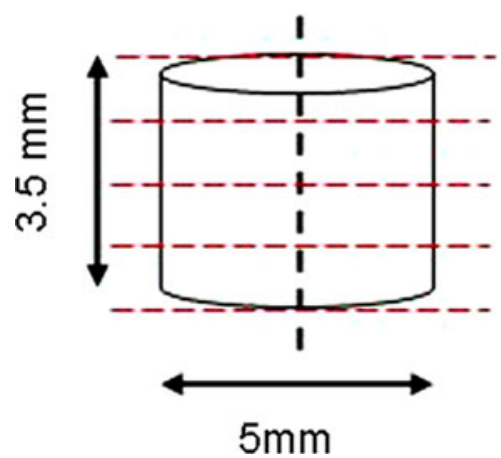

FIG. 1. Dimensions of the original defect; the dotted vertical line represents the sagittal cutting plane, through which explants were cut in half. One of the resultant semicircular defects was used for microCT analysis. The dotted red lines represent a microCT scanning plane (18 $\mu \mathrm{m}$ each).

accumulation and sGAGs. Hence, high X-ray attenuation readings (corresponding to high concentrations of Hexabrix) would therefore indicate regions of sGAG depletion. ${ }^{33,34}$

Several volumes of interest (VOIs) were selected in the bone and overlying articular cartilage regions of each biopsy. For the bone reconstructions, the VOI matched the original cylindrical defect dimensions (divided in half, Fig. 1) and the adjacent native bone (Scanco Medical AG, Brüttisellen, Switzerland). For the articular cartilage reconstructions, the VOI contained the overlying soft tissue of the experimental defects and directly neighboring cartilage, or the "healthy" cartilage samples taken from a larger distance. Next, bone or cartilage three-dimensional reconstructions were obtained using different thresholds that allowed segmentation of both tissues. Attenuation values within the bone reconstructions were considered proportional to the local degree of mineralization of bone, being equivalent to the concentration of hydroxyapatite (HA), ${ }^{35}$ and quantified by comparing the attenuation coefficients with reference measures of a phantom containing $0,200,400,600$, and $800 \mathrm{mg} \mathrm{HA} / \mathrm{cm}^{3}$. Within the cartilage, reconstructions the attenuation values were considered proportional to the distribution and average number of sGAGs.

The average bone volume fraction (bone volume/total volume $[\mathrm{BV} / \mathrm{TV}])$, degree of mineralized bone, and cartilage attenuation value were calculated using Scanco software.

\section{Extracellular matrix biosynthesis}

Quantification of the deposition of extracellular sGAGs was only assessed in the 4-month samples. The original implant area was digested in 3\% papain solution buffer overnight at $60^{\circ} \mathrm{C}$. sGAGs were measured using a Blyscan kit according to the manufacturer's protocol (Biocolor LTD, Carrickfergus, United Kingdom). Both samples and native tissue were measured in three different concentrations in duplicate to ensure measurement in the linear range. Results were depicted as the total glycosaminoglycan deposition per gram of tissue, as well as percentage of native tissue to overcome variation in glycosaminoglycans due to difference in implantation site.

\section{Statistics}

Kolmogorov-Smirnov tests were used to determine normalcy of measurements and, if appropriate, their logarithmics. For evaluation of semiquantitative histological scores the Friedman ranked test was performed. Biochemical, mechanical analysis, and microCT scores were evaluated using a univariate ANOVA to determine significant differences between the three groups, taking the implantation site into account as covariate, and also treatment over native tissue analysis was performed to exclude any covariates. All statistical tests used a significance level of $\alpha=0.05$.

\section{Results}

\section{Colony-forming unit assays}

In the SVF group $5 \times 10^{6}$ cells were implanted and a small portion of the remaining cells was used for colony-forming unit assays. From these assays the percentage of ASCs in the SVF could be calculated. In retrospect between $1.0 \times 10^{5}$ and $3.6 \times 10^{5}$ ASCs were implanted.

\section{Macroscopy}

All goats tolerated surgery well, and no adverse effects occurred during and after surgery. Goats partly immobilized in Softcast gradually increased mobilization in the postoperative period. Upon cast removal after 1 month, goats quickly returned to normal gait.

At sacrifice all goats were still in good health, and upon autopsy no organ showed any macroscopic sign of disease. Furthermore, no signs of osteoarthrosis were observed in any of the knees after 1 month, despite the substantial knee joint area covered by the defects. At this time point, all groups had the same gross appearance score (Tables 1 and 2, maximum 8 points [range of motion excluded due to immobilization]), even though defect edges varied from yellow/reddish to whitish/opaque. Defects did not show any inflammatory response due to the implantation of cells and scaffold material.

Table 1. Macroscopic and Microscopic Score of Implanted Constructs

\begin{tabular}{lcccc}
\hline & SVF (n) & ASC (n) & Acellular (n) & $p$-Value (group) \\
\hline Macroscopy & & & & \\
1 month (max 8 points) & $2.7 \pm 0.6(3)$ & $2.3 \pm 0.6(3)$ & $2.5 \pm 0.8(2)$ & 0.37 \\
4 month (max 10 points) & $7.9 \pm 1.2(8)$ & $6.1 \pm 2.0(9)$ & $6.9 \pm 2.0(7)$ & $<0.01$ \\
$p$-value (over time) & $<0.00001$ & $<0.001$ & & 0.37 \\
Microscopy & & & $6.3 \pm 0.4(2)$ & 0.37 \\
1 month (max 21 points) & $6.2 \pm 1.3(3)$ & $10 \pm 4.7(3)$ & $13.4 \pm 3.2(7)$ & 0.07 \\
4 month (max 21 points) & $15.0 \pm 2.7(8)$ & $11.9 \pm 3.1(9)$ & $<0.01$ & \\
$p$-value (over time) & $<0.01$ & 0.05 & & \\
\hline
\end{tabular}

SVF, stromal vascular fraction; ASC, adipose stem cell. 
Table 2. Degree of Microscopic Cartilage and Bone Regeneration After 4 Months

\begin{tabular}{lccc}
\hline & $S V F(\mathrm{n})$ & ASC (n) & Acellular (n) \\
\hline Cartilage & & & \\
Total & 3 & 1 & 0 \\
Partial & 5 & 6 & 2 \\
None & 0 & 2 & \\
Bone & & 1 & 1 \\
Total & 5 & 7 & 5 \\
Partial & 2 & 1 & 1 \\
None & 1 & & \\
\hline
\end{tabular}

However, defects were still transparent with minimal repair tissue filling the defect in all groups (Fig. 2A).

Gross appearance scores after 4 months increased significantly when compared with 1 month (maximum 12 points, Tables 1 and 2) but not significantly among the three groups. However, surfaces generally appeared more whitish/opaque at the trochlear groove than at the medial condyle, thus resembled the native tissue more closely (Fig. 2). All SVF- and ASC-treated groups showed good integration with the native cartilage at the defect margins, although defect borders were still visible. Predominantly at the medial condyle, slightly reduced cartilage thickness was still present in the center of the defect, likely because implant height was calculated to be somewhat below the cartilage surface. Moreover, a few defects in the ASC-treated group $(n=3)$ showed marks of inflammation and concomitant osteoarthritic degeneration (mainly cartilage erosion; see Fig. 2B, inset). Defects in the acellular group also showed signs of regeneration, although overall surfaces appeared more yellow/reddish than in the cell-treated defects with only a thin cartilaginous coverage that did not reach the cartilage surface (Fig. 2).

\section{Histological evaluation and semiquantitative scoring}

After 1 month no scaffold loss had occurred. Moreover, no inflammatory response was noted in either group. With regard to signs of regeneration, no differences were found between the different treatment groups, with the overall regeneration scores being similar (Tables 1 and 2, Fig. 3, $p=0.37$ ). Remarkably, in all groups, remnants of the suture material were visible in the scaffold material. Remnants of the scaffold material were not seen and could not be visualized since no porcine-specific antibody exists that does not cross-react with goat collagen.

After 4 months of implantation, semiquantitative scores for all groups increased significantly compared to the 1month counterparts (Table 1). In the ASC-treated group (Fig. 4A-D) five out of nine defects healed, whereas three out of nine defects suffered from chronic inflammation, hampering subchondral bone regeneration. Cartilage and subchondral bone regeneration was most advanced in the SVF-treated group, followed by the ASC-treated group and acellular control (Table 2). The main difference is thus the frequency with full and partially filled hyaline-like cartilage and subchondral bone. This can be appreciated further from the immunohistochemical data (Fig. 4). These pictures show cartilage and subchondral bone regeneration 4 months after implantation. Differences are seen in both the cartilaginous part and the subchondral bone. The regenerated cartilage in the SVF group is clearly more hyaline than the fibrocartilage counterparts in the ASC and acellular group. Furthermore, cartilaginous tissue is still present in the regenerated subchondral bone (representative of ongoing osteochondral bone formation) of the ASC-treated group and the acellular control, but already absent in the SVFtreated group (Fig. 4B, F, J).

The overall histological score between all groups did not reach significance due to high variability $(p=0.07)$. However, a significant difference was detected between the SVF-treated and ASC-treated group, if all items were ranked and individually scored (Friedman ranked test, $p=0.02$ ). A trend could be found between the SVF and untreated group $(p=0.07)$, whereas no significance was detected between the ASC-treated and untreated group (Supplementary Table S1).
A

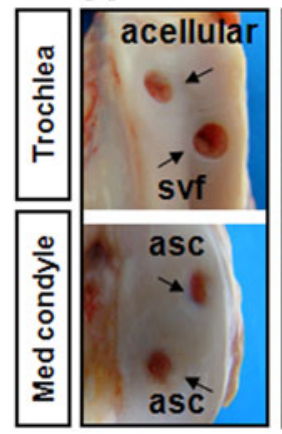

B

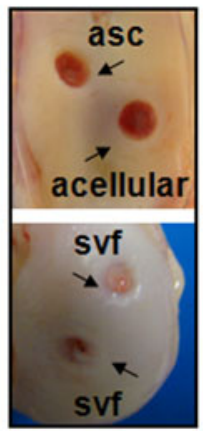

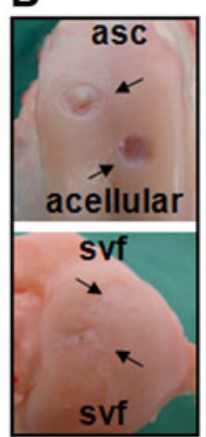
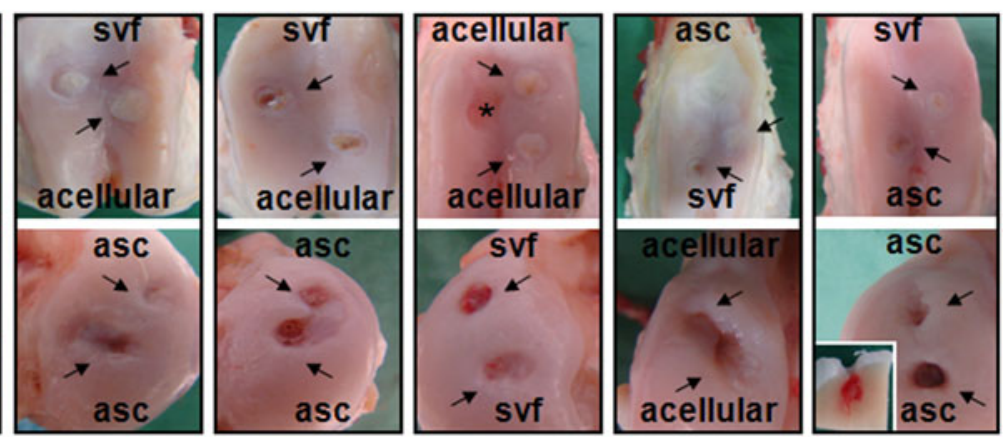

FIG. 2. Gross appearance of all experimental implants. Upper rows represent defects on the trochlea femoris, whereas defects of the medial condyle are depicted in lower rows. Defects are grouped vertically per goat (black rectangles). (A) Implants after 1-month implantation. No obvious differences can be detected between all groups. (B) Implants after 4-month implantation. Implants of all groups showed variable regeneration. Trochlear implants tended to show better regeneration than implants on the medial condyle. The inlay within the last picture shows a longitudinal transsection through an implant suffering from chronic inflammation. No regeneration of the cartilage was observed, but abundant infiltration of blood and inflammatory cells into the subchondral bone were seen. Asterisk marks cartilage defect that was left empty due to faulty burring. 
FIG. 3. Best histological and immunohistochemical staining of ASC-treated (A-C), SVF-treated (DF), and acellular group (G-I) after 1 month of implantation. (A-C) Variable regeneration of the cartilage could be visualized in the ASCtreated group, with little depression, some restoration of the subchondral bone (A), and positive $\mathrm{Col}$ 2 (B) and Alcian Blue (C) staining. (D-F) Some cartilage regeneration was present in the SVF-treated group, as seen by depression of the cartilage, no regeneration of the subchondral bone (D), and faint $\mathrm{Col}$ 2 (E) and Alcian Blue (F) staining. (G-I) There was no regeneration of cartilage and subchondral bone in the acellular group. Bars represent $400 \mu \mathrm{m}$.
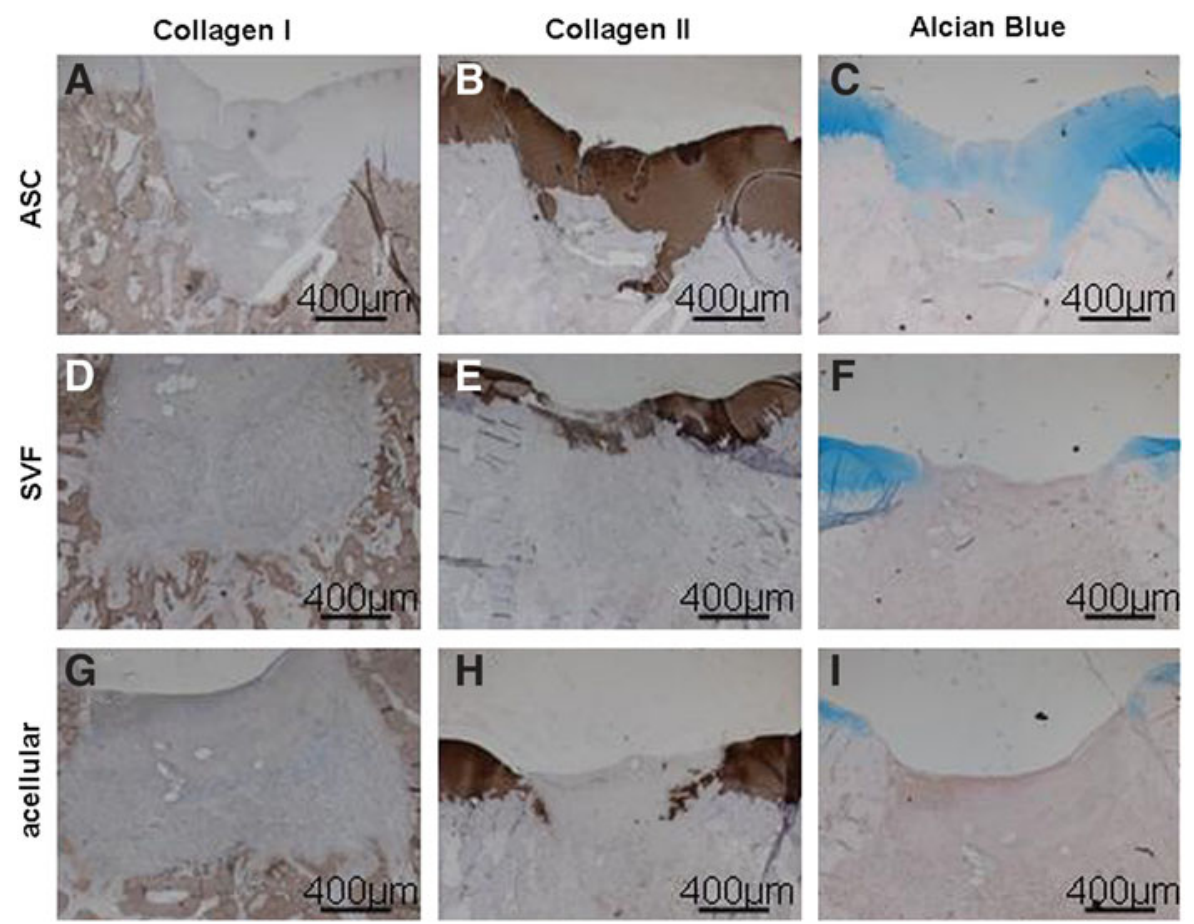

\section{MicroCT analysis}

Implants were analyzed after 4 months for bone and cartilage structure separately, the latter after contrast enhancement using Hexabrix. Recall that the relation between attenuation and proteoglycans is inversely related; that is, the more proteoglycans, the less attenuation (and Hexabrix uptake by the tissue), thus the greener the picture. ASCand SVF-treated groups best resembled native proteoglycan deposition ( $c f$. defect area and adjacent cartilage in Fig. 5A-C).
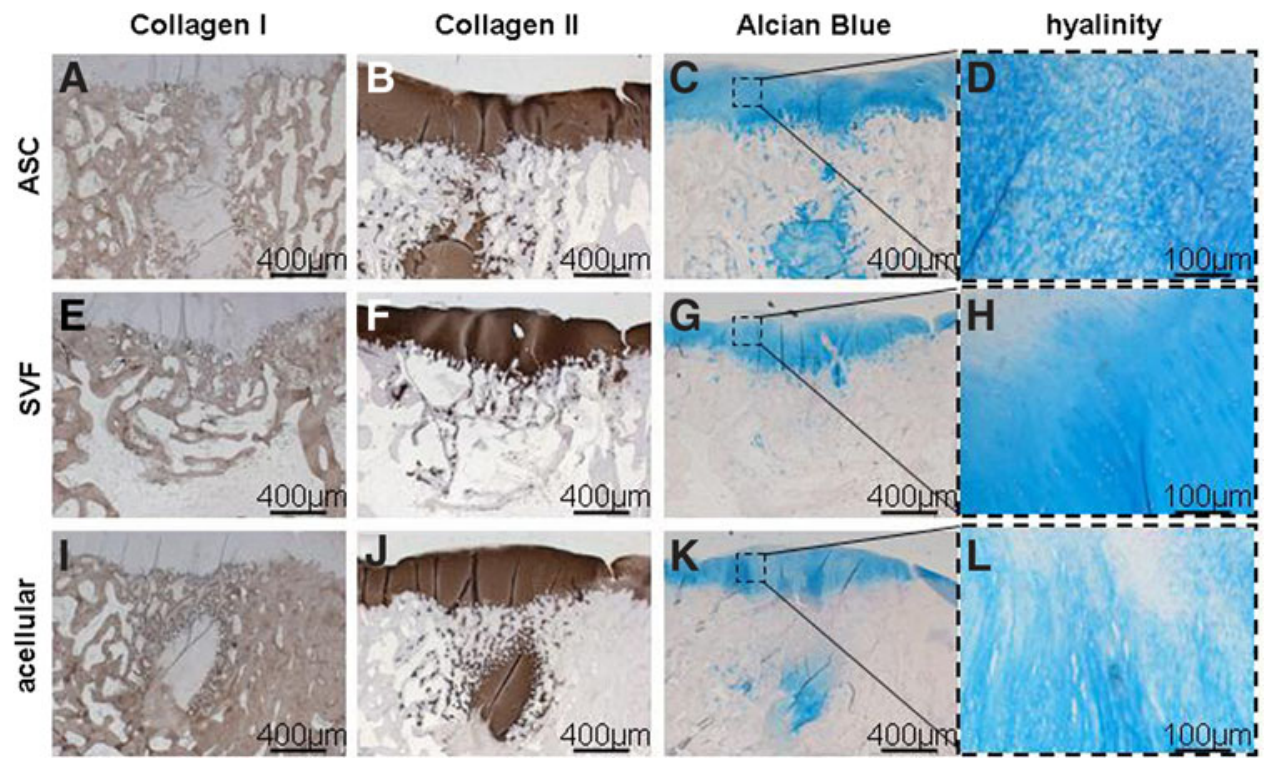

FIG. 4. Representative histological and immunohistochemical staining of ASC-treated (A-D), SVF-treated (E-H), and acellular group (I-L) after 4 months of implantation. (A-D) Clear regeneration of the cartilage could be visualized in the ASC-treated group, with little depression, almost complete restoration of the subchondral bone (A), and abundant Col 2 (B) and metachromatic Alcian Blue (C) staining, but fibrocartilaginous architecture (D). (E-H) Extensive cartilage regeneration was present in the SVF-treated group, as seen by little depression of the cartilage, almost complete regeneration of the subchondral bone (E), abundant Col 2 (F) and metachromatic Alcian Blue (G) staining, and a hyaline-like architecture of the cartilage (H). (I-L) Regeneration of cartilage and subchondral bone in the acellular group varied, with variable depression and subchondral bone regeneration, variable cartilage regeneration, metachromatic matrix staining and a fibrocartilagenous architecture (L). Bars represent $400 \mu \mathrm{m}$. 
A

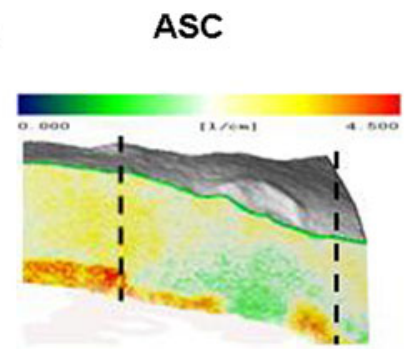

D

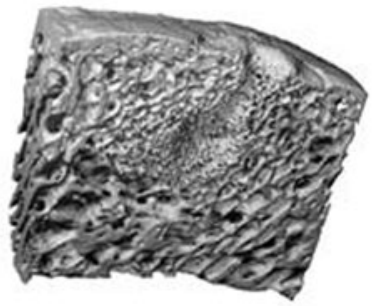

G

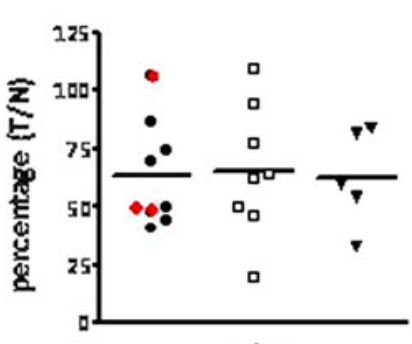

BV/TV
B

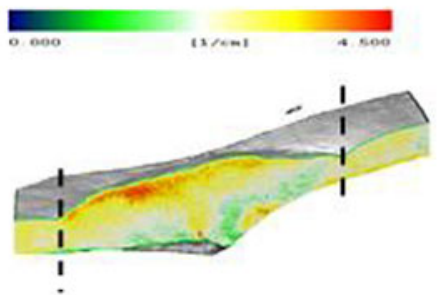

E

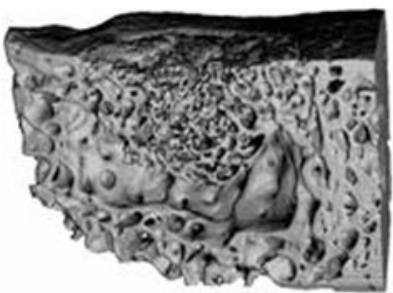

C acellular

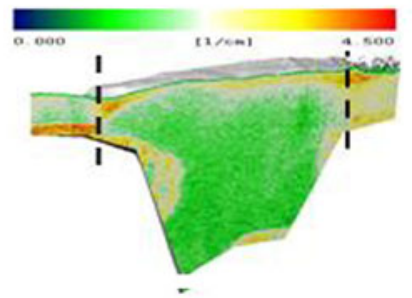

$\mathbf{F}$

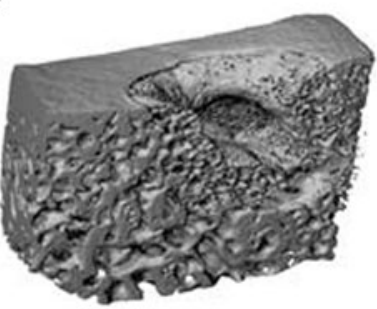

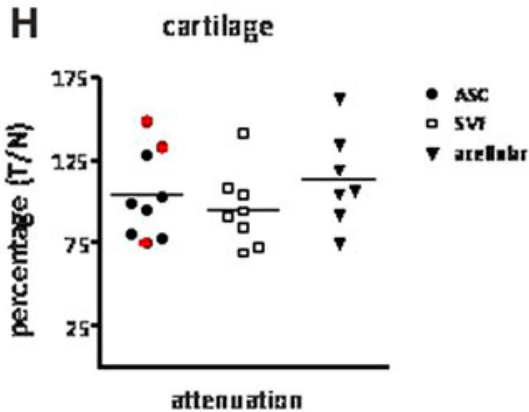

FIG. 5. MicroCT analysis of representative cartilage (A-C) and bone (D-F) explants of the ASC-treated (A, D), SVF-treated $(B, E)$, and acellular (C, F) groups. Colors in explants (A-C) represent cartilage attenuation (the greener, the more proteoglycans). The cartilage in the ASC- and SVF-treated group (between dotted lines indicating defect borders) resembled the adjacent native cartilage more, indicative of better regeneration. These cartilage layers should be seen on top of their subchondral bone underneath. (D-F) ASC- and SVF-treated groups showed more bone regeneration compared to the acellular group, though not significant. $(\mathrm{G}, \mathrm{H})$ Bone and cartilage characteristics of the acellular and SVF- and ASC-treated groups. Results are depicted as treatment over native values $(\mathrm{T} / \mathrm{C})$. All characteristics were in slight favor of the SVF-treated group; however, due to considerable variation in both bone volume to total volume (BV/TV) and cartilage attenuation, no significance was reached. Samples circled in red had chronic inflammation. In the acellular group two values are missing due to technical flaws (G).

However, neither group showed significant differences in attenuation $(p=0.85)$, as can be deduced from Figure $5 \mathrm{H}$.

ASC- and SVF-treated groups tended to show more bone regeneration compared with the acellular group (Fig. 5DF). However, when quantified, no significant difference could be detected in the BV/TV (Fig. 5G), bone maturity (deduced from the number of trabeculae per bone volume), or degree of mineralized bone (Supplementary Table S2).

\section{Extracellular matrix biosynthesis}

After explantation, all samples of the 4-month period were analyzed for sGAG deposition. Determination of sGAG deposition in the 4-month explants showed it to be independent of the implantation site (data not shown). Statistical analysis verified that due to the large heterogeneity in sGAG deposition values in the SVF- and ASC-loaded constructs, one outlier had to be excluded in both cellular groups. No significant differences in sGAG deposition between groups could be detected ( $p=0.65$, Fig. 6).

\section{Mechanical analysis}

Four-month samples were explanted and the functionality of the regenerated cartilage was evaluated by submitting them to a customized indentation test (SVF, $n=9$; ASC, $n=8$; acellular, $n=7$ ). Compressive stress-relaxation represented time-dependent mechanical response to the applied load (Fig. 7C).

A significant difference could be detected between the elastic modulus of the native cartilage of the medial condyle $(1.23 \pm 0.57)$ and trochlear groove $(0.45 \pm 0.14, p<0.05)$. However, no significance was reached in a comparison of the three treated groups, taking into account variation due to difference in implantation site ( $p=0.32$, Fig. 7A). To exclude this variation due to implantation site and each individual goat, treatment over native tissue scores were also compared between the three different groups. Due to large variation, no significance was reached between all groups $(p=0.27$, Fig. 7B). The SVF-treated group contained the largest number of samples approaching native values. 


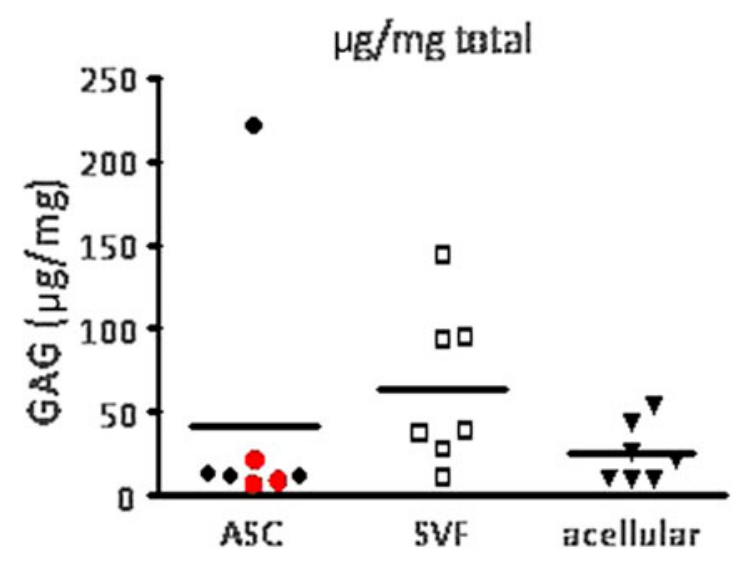

FIG. 6. Glycosaminoglycan (GAG) formation of constructs after 4 months of implantation. GAG synthesis corrected for the weight of tissue. The SVF-treated group showed the highest GAG formation, followed by the ASC-treated group and finally the acellular group. Based on statistical considerations, three samples were excluded as outliers (one in the SVF-treated group, two in the ASC-treated group).

\section{Discussion}

In this pilot study, safety and feasibility of a one-step surgical treatment of focal osteochondral defects in the caprine knee was assessed. Moreover, we compared the regenerative potential of cell-loaded constructs consisting of (1) freshly isolated adipose-derived stromal cells (SVF) with (2) cultured ASCs seeded on collagen type I/III scaffolds and (3) acellular scaffolds. No adverse effects were noted in any group. Tissue repair was rapid, and after 4 weeks the first signs of regeneration could already be observed (ASCs $>$ SVF $>$ acellular, no significant difference), as visualized by immunohistological analyses. After 4 months the regenerative process had clearly progressed, based on immunohistological, biochemical, microCT, and biomechanical parameters. Remarkably, the SVF-treated group now tended to show better regeneration than the ASC-treated group, indicating a catch-up phenomenon of the SVF group. The better result for the ASC group compared with the SVF group at the 4-week time point may be due to lower initial stem cell numbers implanted in the SVF group (in retrospect ranging from $1.0 \times 10^{5}$ to $3.6 \times 10^{5}$ ASCs based on CFU-f assays) compared with the standardized $5 \times 10^{5}$ cultured ASCs in the ASC group. The SVF catch-up phenomenon observed after 4 months may be attributed to other cell types residing in the SVF, such as lymphocytes, pericytes, and vascular smooth muscle cells, possibly leading to synergistic effects on the regenerative process at later stages. An alternative explanation may be that noncultured cells show a higher differentiation potential as cultured cells, as repeatedly reported in other studies. ${ }^{36-38}$ Together, these data suggest that for optimal cartilage regeneration, application of SVF may be favored over cultured ASCs.

As already indicated, we implanted collagenous scaffolds seeded with $5.0 \times 10^{5}$ ASCs or $5.0 \times 10^{6}$ SVF cells (the latter based on our previous studies expected to contain $\sim(1-$ 5) $\times 10^{5}$ ASC-like cells. ${ }^{14}$ This collagen I/III scaffold, containing oriented pores, was previously tested in rabbits ${ }^{39}$ and has already shown promising short- and mid-term results in the clinical setting in combination with chondrocytes to

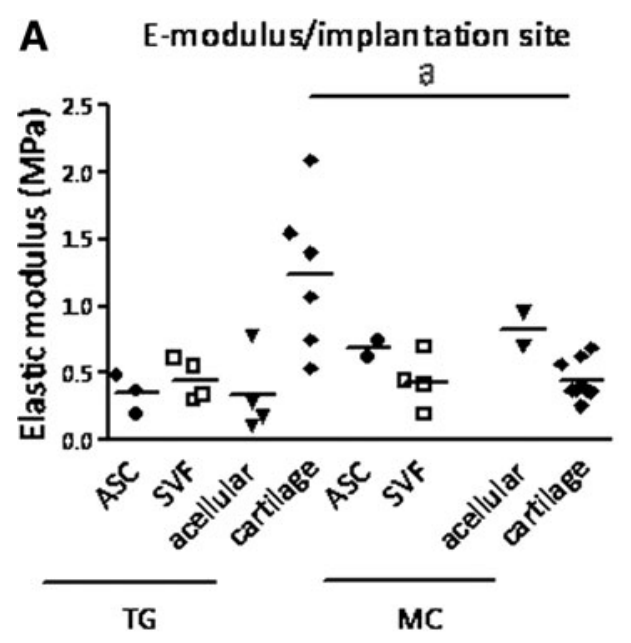

B $\%$ of adjacent native tissue
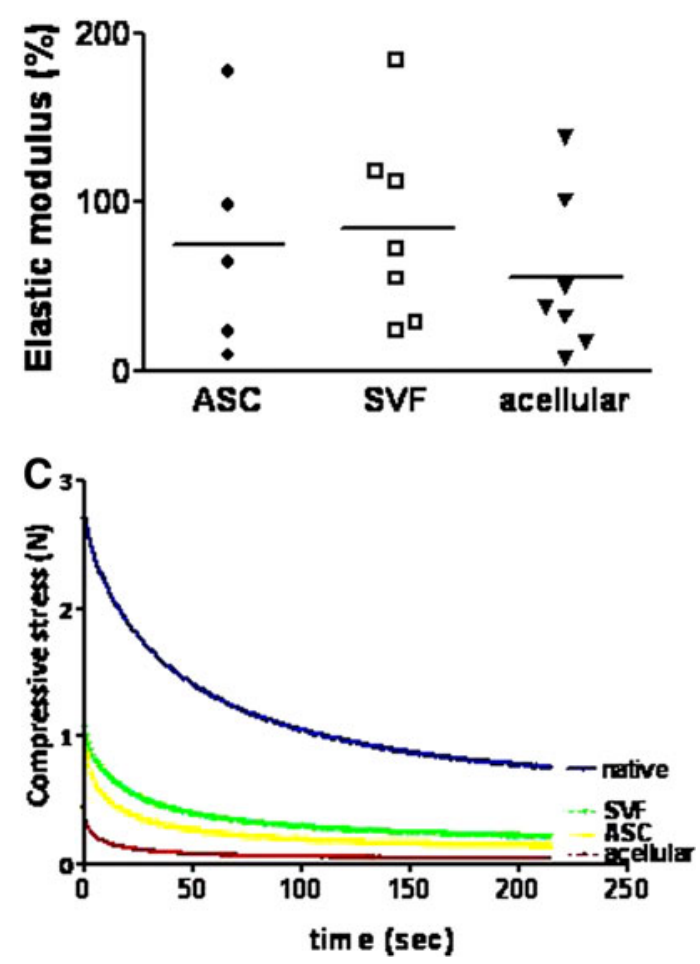

FIG. 7. Mechanical properties of constructs after indentation testing. (A) Elastic modulus of the constructs per implantation site. A large spreading in elastic modulus between the different samples can be observed. A significant difference could be detected between the elastic modulus of the native tissue from the medial condyle and trochlear groove (marked with "a"). (B) Elastic modulus corrected to native tissue. The SVF-treated group again resembled the native tissue best, followed by the ASC-treated group and the acellular group being last. Both in the SVF-treated group as the ASC treated group 1 sample is missing due to technical flaws; moreover, three samples are missing in the ASC-treated group due to inflammation. (C) Representative example of mechanical response to indentation $(200 \mu \mathrm{m})$ showing stress-relaxation curve of the three constructs and native tissue of the trochlear groove. Native tissue showed highest stress peaks, followed by the SVF- and ASC-treated group; the acellular groups showed the lowest stress peak. 
regenerate chondral defects. ${ }^{40}$ Since we seeded our stem cell preparations according to the "dropping on" method ${ }^{41}$ to the upper side of the scaffold, we assume that most of the applied cells would remain in the upper (chondral) part of our scaffold. Since the total volume of the chondral part of the defect was about $20 \mathrm{~mm}^{3}$, this would imply a cell density of $2.5 \times 10^{4}$ cells $/ \mathrm{mm}^{3}$. This would match well with data on the structural organization of cartilage tissue describing a cellular density of 24,000 cells $/ \mathrm{mm}^{3}$ at the articular surface decreasing 3.5-fold to about 7000 cells $/ \mathrm{mm}^{3}$ at the lower half of the upper radial zone ${ }^{42}$ and with studies emphasizing high density to favor chondrogenesis. ${ }^{43,44}$ Currently it is unclear whether application of even higher cell densities would give better results, and this might certainly be interesting to investigate in future experiments.

The acellular control in fact mimicked the well-known (modified) subchondral drilling or microfracture technique, allowing influx of cells from the underlying bone marrow compartment into the scaffold material. This method is widely used, although there is still controversy about its efficacy, and the quality and functionality of the tissue formed. 2,45,46 Nowadays, many studies aim at accelerated cartilage repair by augmenting the scaffolds with either autologous articular chondrocytes cultured from biopsies of non-weight-bearing local cartilage or mesenchymal stem cells like we did in this study. As already stated, the addition of freshly isolated SVF or cultured ASC into the collagen scaffold contributed to better regeneration of the cartilage and subchondral bone. However, how these cells exert this regenerative effect is yet unsolved and is currently a hot topic in the regenerative medicine field. The cells may themselves differentiate into the desired phenotype, they may recruit and activate local mesenchymal stem cells toward regeneration, or they may reactivate local differentiated cells for this purpose. In other words, the early regenerative effect in both cell-treated groups may be due to the paracrine or trophic effect of the stem cells on residing cells rather than their innate differentiation potential. This paracrine effect of stem cells has also been suggested by others to be more important than the differentiation potential of the cells. ${ }^{4-51}$ This is prompted by studies that either found that only $8 \%-33 \%$ of the defect's cell population arose from the implant itself ${ }^{52,53}$ or, in the case of implanting chondrocytes, had no additional contribution at all. ${ }^{54,55}$ Although the study setup does not allow conclusive statements about the actual mechanism of action of the seeded adipose stromal/stem cells, this study consistently showed a positive influence of the implanted cells on the overall regenerative process.

Retainment of a scaffold material in the defect site has been a major challenge in cartilage repair. If scaffold materials are too stiff, they are easily detached from the surrounding cartilage. ${ }^{56,57}$ Several scaffold anchoring strategies have been employed, such as suturing to the adjacent "healthy" cartilage, and the use of hydrogels or sealant to glue the scaffold material to the defect site. However, suturing trauma may lead to osteoarthitic degeneration of the adjacent cartilage, ${ }^{58}$ and human fibrin sealant $\left(\right.$ Tissucol $^{\circledR}$, Baxter, Utrecht, Netherlands) may cause immune reactions leading to cell death. ${ }^{59}$ Tissue reactions to Tissucol were also observed in our pre-pilot study, but we cannot currently rule out that this could be a result of its cross-species use. Eventually, we avoided scaffold loss by a combination of an osteochondral defect allowing pressfitting of the implanted scaffold, suturing onto adjacent carti- lage, and postoperative immobilization. The latter, achieved by using a special designed Softcast to immobilize the goat knees for 4 weeks, reduced shear to a large extent thus preventing scaffold loss effectively. Actually, by the slight muscle atrophy occurring in this 4-week period, flexion and loading of the knee within the cast increased gradually, which probably facilitated and enhanced scaffold integration and cartilaginous differentiation. Whether this immobilization phase should be added in the clinical phase as well remains to be determined.

Not only the addition of regeneration-competent cells, but also the implantation site may influence the extent of regeneration, as illustrated by the differences in elastic moduli of the medial condyle and trochlear groove native tissues. Probably the biomechanical load might be of critical importance in this regard; that is, the higher the biomechanical stress, the stronger the bone and the thicker the overlying cartilage. ${ }^{60,61}$ As a consequence, the elastic modulus depends on anatomical location and on cartilage thickness as was shown by Athanasiou et al. ${ }^{62}$ already in 1994 . If biomechanical stimuli are prime contributors to the extent of regeneration, this could be an explanation for the wide range of regeneration found between various studies, and this would be an argument for describing results as a percentage of the native tissue to exclude this covariate, as was frequently done in this study. Although this might overcome confounding data, and indentation testing is widely used to analyze the mechanical characteristics, it might be less reliable in thin cartilage, as Fischer-Cripps ${ }^{63}$ warned in an article on limitations of indentation measurements. Taking this into account, (too deep) indentation measurements and concomitant values of the underlying subchondral bone might have resulted in confounding data. These results thus have to interpreted with care.

Limitations of this pilot study were the low number of animals used $(n=8)$, the short follow-up period (maximum 4 months), a relatively low number of implanted cells $\left(5 \times 10^{6}\right.$ SVF cells) and the creation of a relatively small defect (diameter $5 \mathrm{~mm}$ ). Therefore, larger studies creating larger defects with long-term follow-up (maximum 2 years) are required to substantiate these findings.

Taken together, this study shows safety and feasibility of a one-step surgical procedure for osteochondral cartilage regeneration using adipose-derived stromal cells. Moreover, freshly isolated adipose stromal cells are at least equal to cultured ASCs in this procedure to regenerate osteochondral defects. In addition to aforementioned arguments of patient friendliness and cost effectiveness by avoiding a second surgical intervention, the availability of commercial systems for intra-operative processing of adipose tissue, meeting GMP conditions, and lowering the risk of infection instigated by "open" processing and prolonged culturing, are important advantages over the current concepts of tissue engineering strategies using cultured cells.

\section{Acknowledgments}

The authors would like to thank the personnel of the University animal facility for their cooperation during surgeries; Margriet Mullender (Department of Plastic Surgery, VUmc) for help with statistical analysis; Leo van Ruijven (Department of Oral Cell Biology and Functional Anatomy, ACTA) for his technical assistance with microCT analysis; and Albert van der Veen, Micha Paalman, and Hans de Koning 
(Departments of Physics and Medical Technology, VUmc) for help with mechanical analysis. Furthermore, regards to Hans Koppers and Piet Finke (3M, Leiden, The Netherlands) for their help with goat immobilization and Development Hybridoma Bank (Department of Biology, University of Iowa, Iowa City, Iowa) for providing the collagen type II antibody. Finally we would like to thank Matricel (Herzogenrath, Germany) for providing us with the collagen type I/ III scaffolds.

\section{Disclosure Statement}

No competing financial interests exist.

\section{References}

1. Hunziker EB. Articular cartilage repair: basic science and clinical progress. A review of the current status and prospects. Osteoarthritis Cartilage. 2002;10:432-463.

2. Bouwmeester PS, Kuijer R, Homminga GN, Bulstra SK, Geesink RG. A retrospective analysis of two independent prospective cartilage repair studies: autogenous perichondrial grafting versus subchondral drilling 10 years postsurgery. J Orthop Res. 2002;20:267-273.

3. Brittberg M. Cell carriers as the next generation of cell therapy for cartilage repair: a review of the matrix-induced autologous chondrocyte implantation procedure. Am J Sports Med. 2010;38:1259-1271.

4. Peterson L, Minas T, Brittberg M, Nilsson A, Sjogren-Jansson E, Lindahl A. Two- to 9-year outcome after autologous chondrocyte transplantation of the knee. Clin Orthop Relat Res. 2000;(374):212-234.

5. Zuk PA, Zhu M, Mizuno H, et al. Multilineage cells from human adipose tissue: implications for cell-based therapies. Tissue Eng 2001;7:211-228.

6. Gronthos S, Franklin DM, Leddy HA, Robey PG, Storms RW, Gimble JM. Surface protein characterization of human adipose tissue-derived stromal cells. J Cell Physiol. 2001;189:54-63.

7. Varma MJ, Breuls RG, Schouten TE, et al. Phenotypical and functional characterization of freshly isolated adipose tissue-derived stem cells. Stem Cells Dev. 2007;16:91-104.

8. Li WJ, Chiang H, Kuo TF, Lee HS, Jiang CC, Tuan RS. Evaluation of articular cartilage repair using biodegradable nanofibrous scaffolds in a swine model: a pilot study. J Tissue Eng Regen Med. 2009;3:1-10.

9. Schoen I, Rahne T, Markwart A, Neumann K, Berghaus A, Roepke E. Cartilage replacement by use of hybrid systems of autologous cells and polyethylene: an experimental study. J Mater Sci Mater Med. 2009;20:2145-2154.

10. Chung C, Burdick JA. Influence of three-dimensional hyaluronic acid microenvironments on mesenchymal stem cell chondrogenesis. Tissue Eng Part A. 2009;15:243-254.

11. Steinert AF, Palmer GD, Pilapil C, Noth U, Evans CH, Ghivizzani $C$. Enhanced in vitro chondrogenesis of primary mesenchymal stem cells by combined gene transfer. Tissue Eng Part A. 2009;15:1127-1139.

12. Hwang NS, Varghese S, Elisseeff J. Derivation of chondrogenically-committed cells from human embryonic cells for cartilage tissue regeneration. PLoS One. 2008;3:e2498

13. Helder MN, Knippenberg M, Klein-Nulend J, Wuisman PI. Stem cells from adipose tissue allow challenging new concepts for regenerative medicine. Tissue Eng 2007;13:1799-1808.

14. Jurgens WJ, van Dijk A, Doulabi BZ, et al. Freshly isolated stromal cells from the infrapatellar fat pad are suitable for a one-step surgical procedure to regenerate cartilage tissue. Cytotherapy. 2009;11:1052-1064.

15. Jurgens WJ, Kroeze RJ, Bank RA, Ritt MJ, Helder MN. Rapid attachment of adipose stromal cells on resorbable polymeric scaffolds facilitates the one-step surgical procedure for cartilage and bone tissue engineering purposes. J Orthop Res. 2011;29:853-860.

16. Ahern BJ, Parvizi J, Boston R, Schaer TP. Preclinical animal models in single site cartilage defect testing: a systematic review. Osteoarthritis Cartilage. 2009;17:705-713.

17. Frisbie DD, Cross MW, Mcllwraith CW. A comparative study of articular cartilage thickness in the stifle of animal species used in human pre-clinical studies compared to articular cartilage thickness in the human knee. Vet Comp Orthop Traumatol. 2006;19:142-146.

18. Lu ZF, Zandieh DB, Wuisman PI, Bank RA, Helder MN. Differentiation of adipose stem cells by nucleus pulposus cells: configuration effect. Biochem Biophys Res Commun. 2007;359:991-996.

19. Jurgens WJ, Oedayrajsingh-Varma MJ, Helder MN, et al. Effect of tissue-harvesting site on yield of stem cells derived from adipose tissue: implications for cell-based therapies. Cell Tissue Res. 2008;332:415-426.

20. Jackson DW, Lalor PA, Aberman HM, Simon TM. Spontaneous repair of full-thickness defects of articular cartilage in a goat model. A preliminary study. J Bone Joint Surg Am. 2001;83A:53-64.

21. Driesang IM, Hunziker EB. Delamination rates of tissue flaps used in articular cartilage repair. J Orthop Res. 2000;18: 909-911.

22. Moran ME, Kim HK, Salter RB. Biological resurfacing of fullthickness defects in patellar articular cartilage of the rabbit. Investigation of autogenous periosteal grafts subjected to continuous passive motion. J Bone Joint Surg Br. 1992;74:659-667.

23. Niederauer GG, Niederauer GM, Cullen LC Jr, Athanasiou KA, Thomas JB, Niederauer MQ. Correlation of cartilage stiffness to thickness and level of degeneration using a handheld indentation probe. Ann Biomed Eng. 2004;32:352-359.

24. Young AA, Appleyard RC, Smith MM, Melrose J, Little CB. Dynamic biomechanics correlate with histopathology in human tibial cartilage: a preliminary study. Clin Orthop Relat Res. 2007;462:212-220.

25. Cousins WJ, Armstrong RW, Robinson WH. Young's modulus of lignin form a continuous indentation test. J Mater Sci. 1975;10:1655-1658.

26. Athanasiou KA, Rosenwasser MP, Buckwalter JA, Malinin TI, Mow VC. Interspecies comparisons of in situ intrinsic mechanical properties of distal femoral cartilage. J Orthop Res. 1991;9:330-340.

27. O'Driscoll SW, Marx RG, Beaton DE, Miura Y, Gallay SH, Fitzsimmons JS. Validation of a simple histological-histochemical cartilage scoring system. Tissue Eng. 2001;7:313-320.

28. Takahashi S, Oka M, Kotoura Y, Yamamuro T. Autogenous callo-osseous grafts for the repair of osteochondral defects. J Bone Joint Surg Br. 1995;77:194-204.

29. Noguchi T, Oka M, Fujino M, Neo M, Yamamuro T. Repair of osteochondral defects with grafts of cultured chondrocytes. Comparison of allografts and isografts. Clin Orthop Relat Res. 1994;251-258.

30. Wakitani S, Goto T, Pineda SJ, et al. Mesenchymal cell-based repair of large, full-thickness defects of articular cartilage. J Bone Joint Surg Am. 1994;76:579-592.

31. Moojen DJ, Saris DB, Auw Yang KG, Dhert WJ, Verbout AJ. The correlation and reproducibility of histological scoring systems in cartilage repair. Tissue Eng. 2002;8:627-634. 
32. Renders GA, Mulder L, Langenbach GE, Koolstra JH. Contrast-enhanced microCT for assessment of articular cartilage and bone morphology in the jaw joint. Bone 2009;44: s253-s338.

33. Palmer AW, Guldberg RE, Levenston ME. Analysis of cartilage matrix fixed charge density and three-dimensional morphology via contrast-enhanced microcomputed tomography. Proc Natl Acad Sci USA. 2006;103:19255-19260.

34. Xie L, Lin AS, Guldberg RE, Levenston ME. Nondestructive assessment of sGAG content and distribution in normal and degraded rat articular cartilage via EPIC-microCT. Osteoarthritis Cartilage. 2010;18:65-72.

35. Nuzzo S, Lafage-Proust MH, Martin-Badosa E, et al. Synchrotron radiation microtomography allows the analysis of three-dimensional microarchitecture and degree of mineralization of human iliac crest biopsy specimens: effects of etidronate treatment. J Bone Miner Res. 2002;17:1372-138.

36. Madeira A, da Silva CL, dos Santos F, Camafeita E, Cabral JM, Sá-Correia I. Human mesenchymal stem cell expression program upon extended ex-vivo cultivation, as revealed by 2 DE-based quantitative proteomics. PLoS One. 2012;7:e43523.

37. Dhanasekaran M, Indumathi S, Lissa RP, Harikrishnan R, Rajkumar JS, Sudarsanam D. A comprehensive study on optimization of proliferation and differentiation potency of bone marrow derived mesenchymal stem cells under prolonged culture condition. Cytotechnology. 2013;65:187-197.

38. Zaim M, Karaman S, Cetin G, Isik S. Donor age and longterm culture affect differentiation and proliferation of human bone marrow mesenchymal stem cells. Ann Hematol. 2012;91:1175-1186.

39. Willers C, Chen J, Wood D, Xu J, Zheng MH. Autologous chondrocyte implantation with collagen bioscaffold for the treatment of osteochondral defects in rabbits. Tissue Eng. 2005;11:1065-1076.

40. Manfredini M, Zerbinati F, Gildone A, Faccini R. Autologous chondrocyte implantation: a comparison between an open periosteal-covered and an arthroscopic matrix-guided technique. Acta Orthop Belg. 2007;73:207-218.

41. Matricel. Optimaix: the third dimension for your cells. Available at www.matricel.net/en/products/optimaix.html

42. Hunziker EB, Quinn TM, Hauselmann HJ. Quantitative structural organization of normal adult human articular cartilage. Osteoarthritis Cartilage. 2002;10:564-572.

43. Concaro S, Nicklasson E, Ellowsson L, Lindahl A, Brittberg M, Gatenholm P. Effect of cell seeding concentration on the quality of tissue engineered constructs loaded with adult human articular chondrocytes. J Tissue Eng Regen Med. 2008;2:14-21.

44. Troken A, Marion N, Hollister S, Mao J. Tissue engineering of the synovial joint: the role of cell density. Proc Inst Mech Eng H. 2007;221:429-440.

45. Khan WS, Johnson DS, Hardingham TE. The potential of stem cells in the treatment of knee cartilage defects. Knee. 2010;17:369-374.

46. Menche DS, Frenkel SR, Blair B, et al. A comparison of abrasion burr arthroplasty and subchondral drilling in the treatment of full-thickness cartilage lesions in the rabbit. Arthroscopy 1996;12:280-286.

47. Chamberlain G, Fox J, Ashton B, Middleton J. Concise review: mesenchymal stem cells: their phenotype, differentiation capacity, immunological features, and potential for homing. Stem Cells 2007;25:2739-2749.

48. Charriere G, Cousin B, Arnaud E, et al. Preadipocyte conversion to macrophage. Evidence of plasticity. J Biol Chem. 2003;278:9850-9855.
49. Jansen BJ, Gilissen C, Roelofs H, et al. Functional differences between mesenchymal stem cell populations are reflected by their transcriptome. Stem Cells Dev. 2009;19:481-490.

50. Mitchell JB, McIntosh K, Zvonic S, et al. Immunophenotype of human adipose-derived cells: temporal changes in stromal-associated and stem cell-associated markers. Stem Cells 2006;24:376-385.

51. Wu L, Leijten JC, Georgi N, Post JN, van Blitterswijk CA, Karperien M. Trophic effects of mesenchymal stem cells increase chondrocyte proliferation and matrix formation. Tissue Eng Part A. 2011;17:1425-1436.

52. Grande DA, Pitman MI, Peterson L, Menche D, Klein M. The repair of experimentally produced defects in rabbit articular cartilage by autologous chondrocyte transplantation. J Orthop Res. 1989;7:208-218.

53. Zarnett R, Salter RB. Periosteal neochondrogenesis for biologically resurfacing joints: its cellular origin. Can J Surg. 1989;32:171-174.

54. Breinan HA, Minas T, Hsu HP, Nehrer S, Sledge CB, Spector $M$. Effect of cultured autologous chondrocytes on repair of chondral defects in a canine model. J Bone Joint Surg Am. 1997;79:1439-1451.

55. Mierisch CM, Wilson HA, Turner MA, et al. Chondrocyte transplantation into articular cartilage defects with use of calcium alginate: the fate of the cells. J Bone Joint Surg Am. 2003;85-A:1757-1767.

56. Drobnic M, Radosavljevic D, Ravnik D, Pavlovcic V, Hribernik M. Comparison of four techniques for the fixation of a collagen scaffold in the human cadaveric knee. Osteoarthritis Cartilage. 2006;14:337-344.

57. Brehm W, Aklin B, Yamashita T, et al. Repair of superficial osteochondral defects with an autologous scaffold-free cartilage construct in a caprine model: implantation method and shortterm results. Osteoarthritis Cartilage. 2006;14:1214-1226.

58. Hunziker EB, Stahli A. Surgical suturing of articular cartilage induces osteoarthritis-like changes. Osteoarthritis Cartilage. 2008;16:1067-1073.

59. van Susante JL, Buma P, Schuman L, Homminga GN, van den Berg WB, Veth RP. Resurfacing potential of heterologous chondrocytes suspended in fibrin glue in large full-thickness defects of femoral articular cartilage: an experimental study in the goat. Biomaterials. 1999;20:1167-1175.

60. Andriacchi TP, Koo S, Scanlan SF. Gait mechanics influence healthy cartilage morphology and osteoarthritis of the knee. J Bone Joint Surg Am. 2009;91(Suppl 1):95-101.

61. Koo S, Gold GE, Andriacchi TP. Considerations in measuring cartilage thickness using MRI: factors influencing reproducibility and accuracy. Osteoarthritis Cartilage. 2005;13:782-789.

62. Athanasiou KA, Agarwal A, Dzida FJ. Comparative study of the intrinsic mechanical properties of the human acetabular and femoral head cartilage. J Orthop Res. 1994;12:340-349.

63. Fischer-Cripps AC. Critical review of analysis and interpretation of nanoindentation test data. Surf Coat Technol. 2011; 200:4153-4165.

Address correspondence to: Marco N. Helder, MSc, PhD Department of Orthopedic Surgery VU University Medical Center De Boelelaan 1117 1081 HV Amsterdam The Netherlands

E-mail: m.helder@vumc.nl 